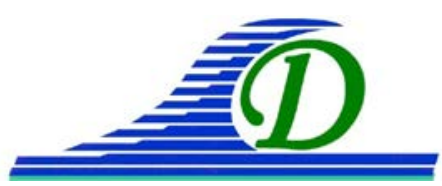

XIII ${ }^{\text {èmes }}$ Journées Nationales Génie Côtier - Génie Civil

Dunkerque, 2-4 juillet 2014

DOI:10.5150/jngcgc.2014.050 (C) Editions Paralia CFL

disponible en ligne - http://www.paralia.fr - available online

\title{
Application d'un modèle d'équilibre dynamique du trait de côte à une plage méso à macrotidale soumise aux houles très énergétiques
}

\author{
Vincent MARIEU ${ }^{1}$, Bruno CASTELLE $^{1}$, Stéphane BUJAN $^{1}$ \\ 1. Université de Bordeaux ; CNRS, UMR 5805-EPOC, avenue de facultés, Talence \\ F-33405, France.v.marieu@epoc.u-bordeaux1.fr
}

\section{Résumé :}

Un modèle d'équilibre de trait de côte est développé et appliqué avec succès à la plage méso à macrotidale du Truc Vert (Aquitaine). Le modèle permet de simuler avec fidélité l'évolution du trait de côte sur ces 8 dernières années avec des temps de calcul très faibles. Par extension, ce concept de modèle d'équilibre dynamique du trait de côte peut être étendu à un modèle d'équilibre dynamique de profil de plage, permettant alors d'observer l'effet de l'historique de l'énergie de la houle sur tout le profil intertidal. Cette approche de modélisation très simple et efficace permet également d'expliquer pourquoi les premières tempêtes d'hiver ont un impact plus important sur l'érosion que les suivantes. Un autre résultat important est que, alors que les processus cross-shore étaient supposés impacter la variabilité du trait de côte sur des échelles de temps courtes (i.e. à l'échelle d'une tempête jusqu'à l'échelle saisonnière), ceux-ci cascadent vers les grandes échelles et permettent d'expliquer les variabilités de la position du trait de côte à l'échelle pluriannuelle.

Mots-clés : Plages sableuses, Erosion, Trait de côte, Dynamique cross-shore, Energie d’équilibre.

\section{Introduction}

La variabilité des côtes sableuses sur des échelles quotidiennes à saisonnière est essentiellement due aux processus cross-shore qui sont contrôlés par l'énergie des vagues incidentes. Sur la plupart des plages ouvertes, les processus longshore ont un effet sur des échelles de temps plus importantes et ne sont pas prépondérants dans la dynamique de la plage à l'échelle annuelle (e.g. YATES et al., 2009). De manière générale, le trait de côte recule rapidement pendant les évènements énergétiques et avance lentement pendant les épisodes de temps calme. Simuler correctement les taux d'érosion et d'accrétion est un défi scientifique. L'utilisation de modèles numériques basés sur une description fine des processus montre qu'il faut systématiquement calibrer le modèle pour chaque événement, la physique n’étant pas complètement bien appréhendée dans ce type de modèles. De plus, l'utilisation de ces modèles pour des simulations à l'échelle annuelle ou interannuelle est encore utopique à l'heure actuelle, du fait des temps de calcul et surtout parce que l'accumulation dans le temps des erreurs 


\section{Thème 2 - Dynamique sédimentaire}

de ces modèles très non-linéaires conduit à des évolutions morphologiques complètement fantaisistes à moyen terme. Des résultats satisfaisants ont été obtenus récemment pour l'évolution du profil de plage sur des échelles de temps de quelques heures à quelques mois (KURIYAMA, 2012), cependant ces modèles ne sont actuellement pas capables de simuler l'évolution de la plage aérienne, en particulier du fait qu'ils ne prennent pas en compte les processus de jet de rive. C'est en partant de ce constat qu'un grand nombre de modèles d'évolution du trait de côte basés sur des lois de comportement ont été développés récemment, la physique fine du système étant filtrée et directement englobée dans le comportement à grande échelle.

Les modèles d'équilibre dynamique sont des modèles de comportement dont l'hypothèse fondamentale est que l'évolution du trait de côte n'est pas uniquement contrôlée par l'énergie des vagues à chaque instant, mais aussi par l'historique de cette énergie. En fonction de l'approche utilisée pour prendre en compte l'historique, ces modèles sont récemment parvenus à simuler avec succès l'évolution du trait de côte durant plusieurs années sur des plages majoritairement micro-tidales (e.g. YATES et al., 2009 ; DAVIDSON et al., 2013 ; SPLINTER et al., 2013). Notre étude montre la première application de ce type de modèle à une plage méso à macrotidale, permettant d'étendre le concept de modèle d'équilibre de trait de côte à tout le profil de plage, de définir le meilleur proxy de trait de côte sur la plage du Truc Vert, et d'expliquer la variabilité pluriannuelle de la plage.

\section{Site d'étude et données}

\subsection{Site d'étude}

La plage du Truc Vert est située sur la côte Aquitaine, une dizaine de kilomètres au nord de la flèche du Cap-Ferret (figure 1a). Elle est constituée de sable fin à moyen $\left(d_{50}=350-400 \mu \mathrm{m}\right)$ et est soumise à un régime de marée méso- macro-tidal, de marnage maximal $4.8 \mathrm{~m}$, et à des houles énergétiques de hauteur significative moyenne $1.36 \mathrm{~m}$ et de période moyenne $6.5 \mathrm{~s}$ de provenance dominante ONO (BUTEL et al. 2002). La variabilité saisonnière de la houle est importante, avec des vagues plus courtes de NO l'été et des vagues plus longues et plus énergétiques d'O à NO, l'hiver. La hauteur significative des vagues peut atteindre $10 \mathrm{~m}$ occasionnellement, et leur période dépasse fréquemment $15 \mathrm{~s}$. La morphologie de la plage est fortement tridimensionnelle et très dynamique, avec deux barres, la barre externe subtidale formant la plupart du temps des croissants d'environ $700 \mathrm{~m}$ de longueur d'onde et la barre interne présentant en général une structure de type barre/chenal (SENECHAL et al., 2009).

\subsection{Données de houle}

Huit ans de données de houle continues toutes les 3 heures ont été nécessaires à la simulation (d'avril 2005 à mars 2013). Elles ont été obtenues à partir de sorties globales 


\section{XIII ${ }^{\text {èmes }}$ Journées Nationales Génie Côtier - Génie Civil \\ Dunkerque, 2-4 juillet 2014}

du modèle WaveWatch III de la NOAA (http://polar.ncep.noaa.gov/waves), au point de grille $\left(2.5^{\circ} \mathrm{O}, 44.5^{\circ} \mathrm{N}\right)$ montré figure $1 \mathrm{a}$. Le biais dans les hauteurs significatives données par le modèle a été corrigé à partir de l'ensemble des 5 ans de données acquises sur la période par la bouée houlographe Candhis 03303 située au large de l'embouchure du bassin d'Arcachon (EPOC/CETMEF http://candhis.cetmef.developpementdurable.gouv.fr). Voir CASTELLE et al. (2014) pour plus d'informations.
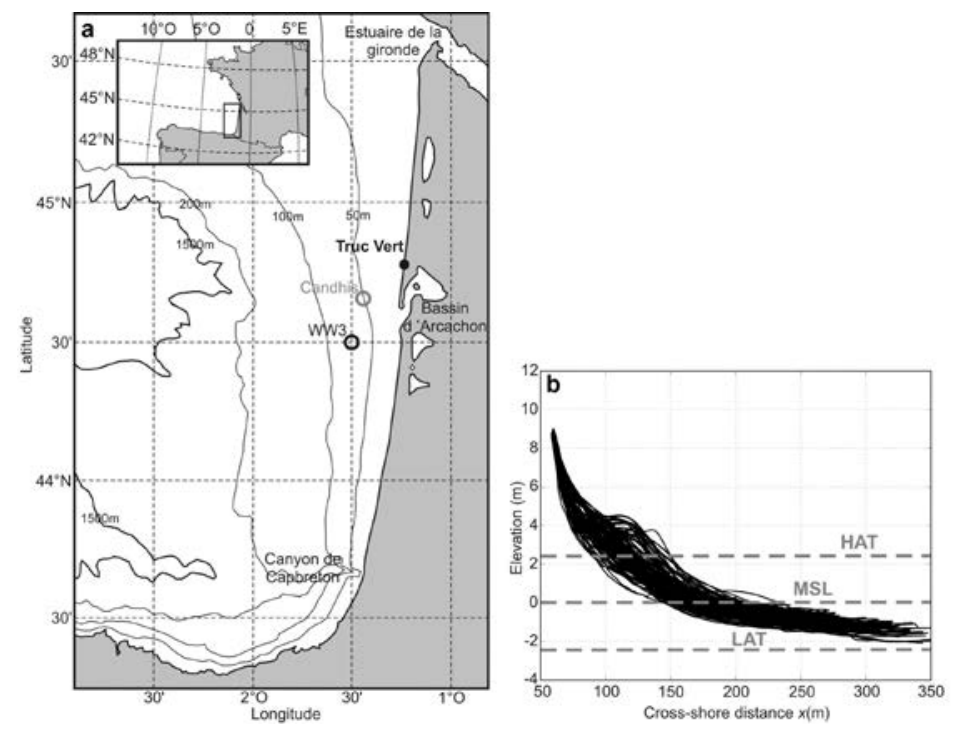

Figure 1. (a) Carte de la côte Aquitaine montrant les emplacements (point noir) de la plage du Truc Vert, (cercle gris) du houlographe Candhis et (cercle noir) du point de grille du modèle WW3 utilisé comme houlographe virtuel. (b) Profils de plage moyens mesurés entre avril 2005 et mars 2013, les niveaux en pointillés correspondent à (HAT)

la plus haute marée astronomique, (MSL) le niveau moyen des mers et (LAT) la plus basse marée astronomique.

\subsection{Topographie de la plage}

Des relevés topographiques de la plage sont réalisés depuis septembre 2003 à une fréquence bimensuelle, lors des marées basses de vive-eau, avec une interruption d'un an en 2008. Les relevés sont effectués au quad, avec un DGPS cinématique Trimble 5700 dont la précision absolue est de $2.5 \mathrm{~cm}$ sur la position horizontale et environ $10 \mathrm{~cm}$ sur la verticale. L'acquisition est effectuée par des transects cross-shore séparés de 20 à $40 \mathrm{~m}$. La distance longshore couverte entre 2003 et 2008 était de $350 \mathrm{~m}$ mais elle a été étendue à 750 m à partir de 2008 puis à 1200 m à partir de 2012, pour s'affranchir au maximum de la variabilité longshore due aux structures 3D. Ces données sont ensuite interpolées par une méthode de krigeage anisotrope sur une grille de résolution $2 \mathrm{~m} \times 20$ $\mathrm{m}$ dans les directions cross-shore et longshore, respectivement. Chaque grille obtenue a été moyennée pour obtenir un profil de plage moyen, associé à un écart type représentant la variabilité longshore de la portion de plage relevée (figure 1b). Il est 


\section{Thème 2 - Dynamique sédimentaire}

indispensable d'utiliser une méthode d'interpolation anisotrope, les méthodes isotropes générant des oscillations non physiques entre les transects, conduisant à un profil moyen lissé au niveau de la berme et de la barre intertidale.

\section{Modèle d'équilibre de plage}

Du fait des faibles gradients de transport sédimentaire longshore au niveau de la plage du Truc Vert (IDIER et al., 2013), la variabilité du trait de côte est essentiellement due aux processus cross-shore rendant pertinente l'application d'un modèle d'équilibre. Le modèle de YATES et al. (2009) a été appliqué sur la plage du Truc Vert, il est basé sur le principe d'équilibre de plage, c'est à dire que pour une position donnée du trait de côte, $S$, il existe une énergie d'équilibre théorique des vagues $E_{e q}$ pour laquelle la position du trait de côte ne bouge pas. Le modèle décrit alors le mouvement du trait côte comme étant fonction à la fois de l'écart à cette énergie d'équilibre et de l'énergie des vagues elle-même :

$\frac{d S}{d t}=C^{ \pm} E^{1 / 2}\left(E-E_{e q}\right)$

où $E$ est l'énergie des vagues, et $C^{+}$et $C^{-}$sont respectivement des coefficients d'accrétion (quand $E-E_{e q}<0$ ) et d'érosion (quand $E-E_{e q}>0$ ). Le modèle de YATES et al. (2009) suppose que l'énergie d'équilibre est une fonction affine de la position du trait de côte :

$E_{e q}(S)=a S+b$

où $a$ et $b$ sont des paramètres libres du modèle.

Afin de déterminer les coefficients du modèle, une optimisation non linéaire par la méthode du Recuit Simulé a été utilisée (BERTSIMAS \& TSITSIKLI, 1993), les paramètres optimaux étant ceux qui permettent de minimiser la somme des écarts entre les positions des trait de côte calculée et mesurée lors de chaque relevé. Un cinquième paramètre, $d$, a été utilisé pour tenir compte d'une possible erreur sur la première mesure. En effet, cette dernière servant de point de départ à la simulation, l'erreur de mesure aurait un impact trop important sur l'évolution simulée de la position du trait de côte. Ce modèle a été appliqué pour différents proxys de trait de côte définis par la position cross-shore des iso-contours d'altitudes $-1 \mathrm{~m}$ à $8 \mathrm{~m}$ par rapport au niveau moyen des mers, avec un pas de $0.1 \mathrm{~m}$. Les coefficients du modèle ont été calculés sur la période de 8 ans de mesures.

\section{Résultats et discussion}

\subsection{Evolution et proxy de trait de côte}

La figure 2 montre les résultats de la simulation de l'évolution du profil de plage durant la période de 8 ans de mesures. Pour tous les proxys de trait de côte, $-1 \mathrm{~m}<z<8 \mathrm{~m}$, la variabilité saisonnière est très importante, avec une lente accrétion durant les périodes 


\section{XIII ${ }^{\text {èmes }}$ Journées Nationales Génie Côtier - Génie Civil \\ Dunkerque, 2-4 juillet 2014}

de vagues peu énergétiques et une érosion rapide durant les évènements énergétiques. Comme souligné par YATES et al. (2009), les premières tempêtes d'hiver (décembre janvier) sont responsables des érosions les plus importantes, les tempêtes suivantes (février - mars), bien que très énergétiques, n’érodant pas la plage significativement (figure 2b). Ceci est dû au fait que l'écart entre l'énergie des premières tempêtes et l'énergie d'équilibre de la plage à son pic d'accrétion est très important (figure 2a). Par exemple, ce phénomène est particulièrement visible à la fin de l'année 2009, le premier évènement énergétique est responsable de presque la totalité de l'érosion hivernale bien que les évènements du début de l'année 2010 soient d'amplitude équivalente. Cela explique également pourquoi les tempêtes observées durant la campagne de mesure ECORS 2008 (SENECHAL et al., 2011) n’ont pas significativement érodé la plage, celle-ci ayant déjà été érodée par une tempête précédente, générant une énergie d'équilibre plus importante. A l'inverse, même si l'énergie des vagues est encore élevée au début du printemps, la plage recommence généralement à engraisser, du fait que l'énergie d'équilibre est très importante.

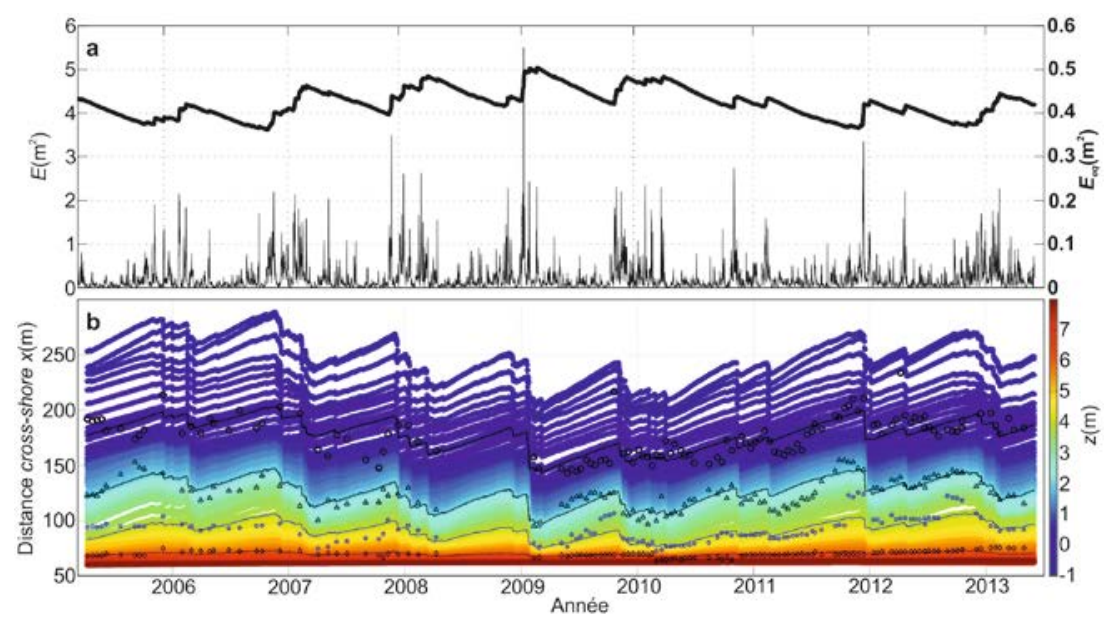

Figure 2. (a) Energie observée (trait fin) and énergie d'équilibre calculée (trait gras) pour le proxy de trait de côte $\mathrm{z}=2 \mathrm{~m}$. (b) Evolution de la position du trait de côte simulée pour tous les proxys de trait de côte $-1 m<z<8 m$ à $0.1 \mathrm{~m}$, avec l'évolution de la position du trait de côte pour les 4 proxys $z=0 \mathrm{~m}, 2 \mathrm{~m}, 4 \mathrm{~m}$ et $6 \mathrm{~m}$ et les mesures correspondantes ajoutées en noir.

Les performances du modèle ne sont pas homogènes sur tous les proxys de trait de côte. Un calcul du coefficient de détermination $R^{2}$ et de l'erreur quadratique moyenne RMSE a été effectué pour chaque proxy de trait de côte (voir CASTELLE et al. 2014 pour plus de détails), montrant que le modèle affiche des bonnes performances pour $1 \mathrm{~m}<\mathrm{z}<4$ $\mathrm{m}$, avec une erreur minimale aux alentours de $z=1.5 \mathrm{~m}\left(R^{2}=0.65\right.$ et $\left.R M S E=8 \mathrm{~m}\right)$. Aux altitudes $z<1 \mathrm{~m}$ et $\mathrm{z}>4 \mathrm{~m}$, les performances du modèle sont significativement dégradées; en effet, la zone $z<1 \mathrm{~m}$ est influencée par la dynamique des barres 


\section{Thème 2 - Dynamique sédimentaire}

intertidales et la zone $z>4 \mathrm{~m}$ par celle de la berme. Ce résultat indique que le meilleur proxy de trait de côte pour la plage du Truc Vert est l'iso-ligne $z=1.5 \mathrm{~m}$, ce qui correspond au niveau moyen des hautes mers. Ce résultat pourrait avoir une incidence sur les études basées sur la l'imagerie vidéo, pour lesquelles le niveau de la marée haute de morte-eau est généralement utilisé car il permet d'obtenir des données quotidiennes ( $z=0.4 \mathrm{~m}$ au Truc Vert).

\subsection{Variabilité des paramètres sur le profil de plage}

Du fait que la plage du Truc-Vert est meso- macro-tidale, les paramètres $a, b, C^{+}$et $C^{-}$ des équations (1) et (2) ont été recherchés séparément pour chacun des proxys de trait de côte. La figure 3 montre la variabilité verticale de ces paramètres sur le profil de plage, ce qui souligne les différences de comportement en fonction des zones du profil considérées. La variabilité verticale des différents paramètres est très importante et les courbes montrent une bonne cohérence pour $0 \mathrm{~m}<z<4 \mathrm{~m}$. Pour $z>4 \mathrm{~m}$, le modèle n'est plus adapté, cela s'explique par le fait que la morphologie du haut de plage n'est directement influencé par les vagues que lors des tempêtes très énergétiques combinées à des marnages importants. On atteint ici les limites du modèle, qui bénéficierait de la prise en compte du marnage. Pour $z<0 \mathrm{~m}$, les effets de la dynamique de la barre interne et le fait que la pente de plage y soit très faible génèrent également moins de cohérence dans l'évolution des paramètres. L'augmentation de $a$ avec $z$ (figure 3a) signifie que l'énergie des vagues a une influence à beaucoup plus long terme sur le haut de plage que sur le bas de plage qui répond de manière plus instantané à l'énergie des vagues. Le paramètre $b$ dépend de la moyenne temporelle de la position du trait de côte et a donc moins de signification physique. De même que $a, C$ décroit avec z (figure 3d), indiquant que le trait de côte est érodé moins rapidement en haut de plage qu'en bas de plage pour une énergie de vagues donnée. L'évolution de la vitesse d'accrétion $C^{+}$est plus surprenante (figure 3c), celle-ci présentant un pic à $z=3 \mathrm{~m}$. Cela peut être expliqué par le fait qu'il s'agit de la position typique de la berme dont la construction peut être très rapide lors des épisodes d'énergie modérée.

\subsection{Tendance à l'échelle interannuelle}

La figure 4 montre l'évolution du trait de côte pour le proxy $z=1.5 \mathrm{~m}$ sur les 8 années de mesure. Le modèle est capable de reproduire la tendance érosive entre 2006 et 2009 puis la tendance accrétive entre 2009 et 2013. Ce résultat montre que cette tendance qui n’a jamais été expliquée peut être uniquement due à des processus cross-shore tels que ceux pris en compte dans le modèle. Ainsi, les effets cross-shore, jusqu'ici supposés avoir un impact uniquement à court terme, peuvent décrire des tendances à long terme par un effet de cascade des petites vers les grandes échelles spatio-temporelles. Ce résultat semble être confirmé par les récentes mesures du début d'année 2014 au Truc Vert (non montré ici). 


\section{XIII ${ }^{\text {èmes }}$ Journées Nationales Génie Côtier - Génie Civil \\ Dunkerque, 2-4 juillet 2014}
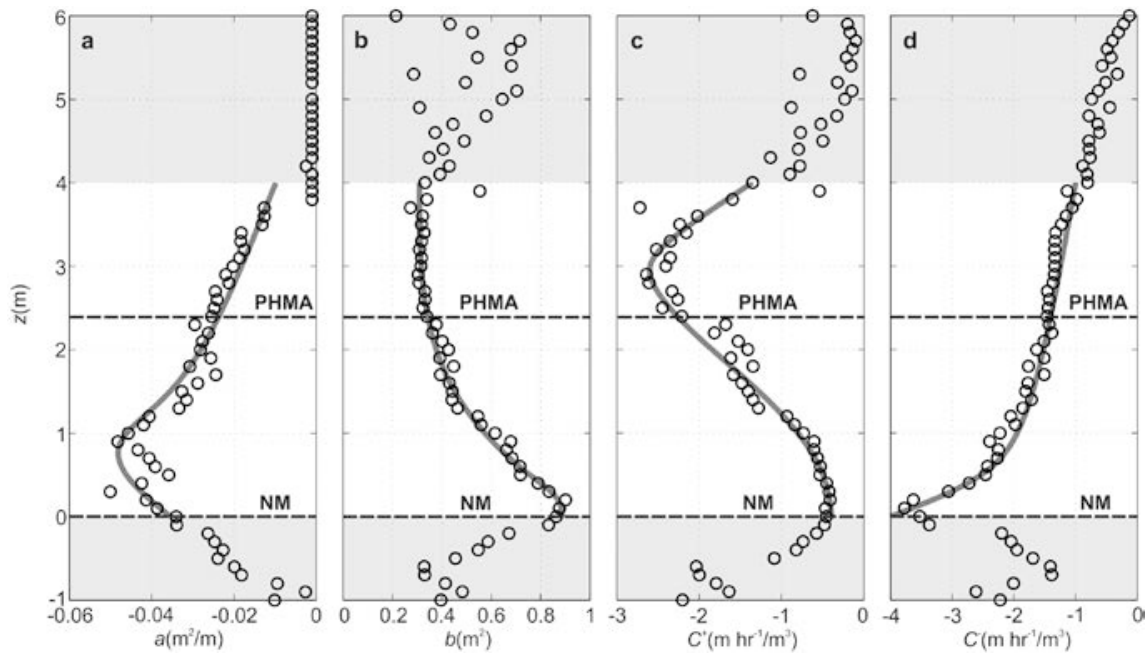

Figure 3. Paramètres du modèle en fonction du proxy z de trait de côte : (a) pente d'équilibre $a,(b)$, ordonnée à l'origine de la droite d'équilibre d'énergie des vagues $b$,(c) le coefficient d'accrétion $C^{+}$et (d) le coefficient d'érosion $C^{-}$. Dans chaque sousfigure, les traits pointillés indiquent le niveau moyen des mers et le niveau de la plus haute marée astronomique.

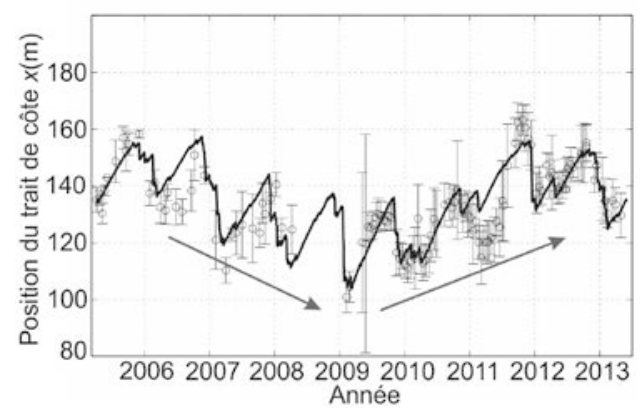

Figure 4. Evolution de la position du trait de côte simulée (trait noir) pour le proxy de trait de côte $\mathrm{z}=1.5 \mathrm{~m}$ durant les 8 années de mesures (cercles gris) sur la plage du Truc Vert. Les barres d'erreur en gris correspondent à la variabilité longshore de la plage. Les flèches soulignent la tendance pluriannuelle.

\section{Conclusions}

Les résultats montrent que le concept de modèle d'équilibre fonctionne sur les plages méso à macrotidales lorsque le proxy de trait côte est choisi correctement. Les simulations de 8 années de levés de la plage du Truc Vert ont montré que ce proxy était l'iso-contour correspondant à la moyenne des hautes mers. Les modèles d'équilibre permettent de montrer que l'effet des tempêtes n'est pas uniquement fonction de leur énergie mais également de l'historique de la plage. Enfin, bien que prenant uniquement en compte des processus cross-shore, ce modèle a permis de simuler des tendances pluriannuelles sur la plage du Truc Vert. Ce modèle va à présent être utilisé pour l'étude 
de l'évolution du trait de côte à l'échelle décennale, en réponse aux changements climatiques.

\section{Références bibliographiques}

BERTSIMAS D., TSITSIKLIS J. (1993). Simulated annealing. Statistical Science, Vol. 8, pp 10-15. http://dx.doi.org/10.1214/ss/1177011077

BUTEL R., DUPUIS H., BONNETON P. (2002). Spatial variability of wave conditions on the French Atlantic coast using in-situ data. Journal of Coastal Research, Vol. SI 36, pp 96-108.

CASTELLE B., MARIEU V., BUJAN S., FERREIRA S., PARISOT J.P., CAPO S., SENECHAL N. CHOUZENOUX T. (2014). Equilibrium shoreline modelling of a highenergy meso-macrotidal multiple-barred beach. Marine Geology, Vol. 347, pp 85-94. http://dx.doi.org/10.1016/j.margeo.2013.11.003

DAVIDSON M.A., SPLINTER K.D. TURNER I.L. (2013). A simple equilibrium model for predicting shoreline change. Coastal Engineering. Vol. 73. pp 191-202. http://dx.doi.org/10.1016/j.coastaleng.2012.11.002

IDIER D., CASTELLE B., CHARLES E., MALLET C. (2013). Longshore sediment flux hindcast: spatiotemporal variability along the SW Atlantic coast of France. Journal of Coastal Research, Vol. SI 65, pp 1785-1790.

KURIYAMA Y. (2012). Process-based one-dimensional model for cyclic longshore bar evolution. Coastal engineering. Vol. 62, pp 48-61.

http://dx.doi.org/10.1016/j.coastaleng.2011.12.001

SENECHAL N. GOURRIOU T., CASTELLE B., (...), HOWA H. (2009). Morphodynamic response of a meso- to macro-tidal intermediate beach based on a long-term dataset. Geomorphology, Vol. 107. pp 263-274.

http://dx.doi.org/10.1016/j.geomorph.2008.12.016

SENECHAL N., ABADIE S., GALLAGHER E., (...), GARLAN T. (2011). The ECORS-Truc Vert'08 nearshore field experiment: presentation of a three-dimensional morphologic system in a macro-tidal environment during consecutive extreme storm conditions. Ocean Dynamics, Vol. 61, pp 2073-2098. http://dx.doi.org/10.1007/s10236-011-0472-x SPLINTER K.D., TURNER I.L., DAVIDSON M.A. (2013). How much data is enough? The importance of morphological sampling and duration for calibration of empirical shoreline models. Coastal Engineering, Vol. 77, pp 14-27. http://dx.doi.org/10.1016/j.coastaleng.2013.02.009

YATES M.L., GUZA R.T., O’REILLY W.C. (2009). Equilibrium shoreline response: observations and modeling. Journal of Geophysical Research, Vol.114, C09014. http://dx.doi.org/10.1029/2009JC005359 